\title{
A fast infrared radiative transfer model for overlapping clouds
}

\author{
Jianguo Niu and Ping Yang \\ Department of Atmospheric Sciences, Texas A\&M University, College Station, TX 77843 \\ Huang-Lung Huang, James E. Davies, and Jun Li \\ Cooperative Institute of Satellite Study University of Wisconsin, Madison, WI 53706 \\ Bryan A. Baum and Yong X. Hu \\ NASA Langley Research Center, Hampton, VA 23681
}

For publication in

J. of Quantitative Spectroscopy \& Radiative Transfer

Corresponding author address: Dr. Ping Yang, Department of Atmospheric Sciences, Texas

A\&M University, College Station, Texas. Email: pyang@ariel.met.tamu.edu 


\begin{abstract}
A fast infrared radiative transfer model (FIRTM2) appropriate for application to both single-layered and overlapping cloud situations is developed for simulating the outgoing infrared spectral radiance at the top of the atmosphere (TOA). In FIRTM2 a pre-computed library of cloud reflectance and transmittance values is employed to account for one or two cloud layers, whereas the background atmospheric optical thickness due to gaseous absorption can be computed from a clear-sky radiative transfer model. FIRTM2 is applicable to three atmospheric conditions: 1) clear-sky, 2) single-layered ice or water cloud, and 3) two simultaneous cloud layers in a column (e.g., ice cloud overlying water cloud). Moreover, FIRTM2 outputs the derivatives (i.e., Jacobians) of the TOA brightness temperature with respect to cloud optical thickness and effective particle size. Sensitivity analyses have been carried out to assess the performance of FIRTM2 for two spectral regions, namely the longwave (LW) band (587.3 $1179.5 \mathrm{~cm}-1)$ and the short-to-medium wave (SMW) band $(1180.1-2228.9 \mathrm{~cm}-1)$. The assessment is carried out in terms of brightness temperature differences (BTD) between FIRTM2 and the well-known discrete ordinates radiative transfer model (DISORT), henceforth referred to as BTD (F-D). The BTD (F-D) values for single-layered clouds are generally less than $0.8 \mathrm{~K}$. For the case of two cloud layers (specifically ice cloud over water cloud), the BTD(F-D) values are also generally less than $0.8 \mathrm{~K}$ except for the SMW band for the case of a very high altitude $(>15 \mathrm{~km})$ cloud comprised of small ice particles. Note that for clear-sky atmospheres, FIRTM2 reduces to the clear-sky radiative transfer model that is incorporated into FIRTM2, and the errors in this case are essentially those of the clear-sky radiative transfer model.
\end{abstract}

Keywords: Infrared radiative transfer; Fast code; Cloud; Single scattering properties of cloud. 


\section{Introduction}

Remote sensing techniques based on hyperspectral measurements provide powerful approaches for retrieving a variety of atmospheric parameters [1] [2]. A forward radiative transfer (RT) model is normally required in combination with measurements to infer cloud microphysical and optical properties. The computational efficiency of a retrieval algorithm based on hyperspectral measurements is, in practice, critical to the usefulness of the retrieval algorithm. Several fast RT models for clear-sky conditions have been developed such as the fast monochromatic model developed by Strow et al. [3]. For cloudy atmospheres, Moncet and Clough [4] developed a fast RT model using the adding/doubling principle. Rathke and Fischer [5] developed a fast RT model based on the two-stream source function technique to compute the upward radiance. Additionally, Rathke and Fischer [6] also assessed several approximate solutions to the infrared (IR) RT equation through comparison with the more rigorous discrete ordinate radiative transfer (DISORT [7] model. Fu et al. [8] discussed various two-stream and four-stream approximations applied to the simulation of thermal infrared radiative transfer and accounted for the effect of multiple scattering.

More recently, Wei et al. [9] presented a fast infrared RT model (hereafter, FIRTM1) for single-layered clouds. In FIRTM1 the background clear-sky optical thickness associated with gaseous absorption is determined from application of the fast clear-sky RT model developed by

Strow et al. [3]. The cloud effects are obtained from a pre-computed look-up table of cloud transmittance and reflectance properties. For liquid water clouds in FIRTM1, Lorenz-Mie theory is employed to derive the optical properties for gamma size distributions of water droplets. For ice clouds, a population of droxtals, pristine hexagonal ice columns and aggregates is assumed in the particle size distributions. There are many approaches available to compute the single- 
scattering properties of non-spherical particles, as reviewed by Mishenchenko et al. [10]. In FIRTM1 the single-scattering properties of individual non-spherical ice particles are derived from the composite method (Fu et al. [11]), which combines the single-scattering properties from the finite-difference time domain method [12-16], improved geometric optics method [17], and Lorenz-Mie theory.

The root mean square (RMS) errors of FIRTM1, measured in terms of brightness temperature differences (BTD) when compared with the more rigorous DISORT model, are typically less than $0.5 \mathrm{~K}$. In terms of computational efficiency, the CPU time required by FIRTM1 is approximately three orders of magnitude less than that required by DISORT for the same atmospheric optical profile.

The FIRTM1 is limited to single-layered clouds, but in reality multilayered clouds are commonly observed. In particular, ice clouds are often seen overlying low-level water clouds. This study accounts for this cloud configuration by presenting a fast infrared radiative transfer model for two cloud layers (hereafter denoted FIRTM2). In terms of methodology, FIRTM2 is a generalization of FIRTM1, as the radiative transfer approach in both models accounts for multiple scattering using pre-calculated look-up tables of cloud reflectance and transmittance properties. The output of FIRTM2 also includes the derivatives (Jacobians) of the brightness temperature corresponding to the TOA radiance with respect to cloud optical thickness and effective particle size. The analytical Jacobians are used to investigate the sensitivity of the IR spectrum to changes in cloud optical and microphysical properties.

This paper is organized as follows. Section 2 presents the theoretical basis for FIRTM2, including the development of the look-up tables of cloud reflectance and transmittance properties. Section 3 presents the differences between FIRTM2 and the DISORT model, which 
serves as a sort of validation exercise for the FIRTM2 accuracy. In Sec. 4, the analytical Jacobians are formulated and used subsequently to investigate the sensitivity to cloud optical properties of the IR spectrum computed from FIRTM2 . Section 5 concludes the study.

\section{Approximate radiative transfer solution for two cloud layers}

Similar to FIRTM1, FIRTM2 is a plane-parallel approximate radiative transfer model for estimating the upward TOA infrared radiance. It can be applied to three atmospheric conditions: 1) clear-sky, 2) one cloud layer (either ice or liquid water), and 3) two cloud layers (e.g., ice cloud overlying a liquid water cloud). In FIRTM2, the atmosphere is divided into 100 layers. The background optical thickness of each layer due to atmospheric gaseous absorption can be computed from an efficient clear-sky RT model. Examples include the model developed by Strow et al. [3], or a line-by-line RT model (LBLRTM). The computational expense of employing a LBLRTM may be prohibitive for some remote sensing problems. For a clear-sky atmosphere, FIRTM2 essentially reduces to the clear-sky RT model incorporated into FIRTM2.

A cloud layer in FIRTM2 can be defined by specifying the cloud optical thickness, which is referenced to a visible wavelength, and the effective particle size. The effective particle size is defined as the ratio of the volume to projected area for a given particle size distribution and is discussed further in the next section. These properties are used to determine the cloud layer transmittance and reflectance characteristics that are provided in a pre-computed look-up table, as will be discussed below. The infrared optical thickness of a cloud, $\tau_{\lambda}$, can be specified as follows:

$$
\tau_{\lambda}=\frac{\left\langle Q_{e}(\lambda)\right\rangle}{2} \tau_{v i s},
$$


where $\tau_{v i s}$ is the value of optical thickness at a visible wavelength of $0.55 \mu \mathrm{m}$, and $\left\langle Q_{e}(\lambda)\right\rangle$ is the mean extinction efficiency at the specified IR wavelength, $\lambda$. In Eq. (1) the mean extinction efficiency for cloud particles at visible wavelengths assumes the value 2 (i.e., the asymptotic value from geometric optics). This assumption is usually valid, particularly for ice clouds since ice particles are typically much larger than a visible wavelength.

As shown in Fig.1, a two-layered cloud system can be regarded as consisting of five regions: the atmosphere between the surface and the lower-level cloud, the layer comprising the lower-level cloud, the atmosphere between the lower- and higher-level clouds, the layer comprising the higher-level cloud, and the atmosphere above the higher-level cloud. The quantities $I_{i}^{\downarrow}$ and $I_{i}^{\uparrow}(i=1-5)$ represent the contributions to the downward and upward thermal radiances from various components of the atmosphere. Specifically, $I_{i}^{\downarrow}$ and $I_{i}^{\uparrow}(i=1,3,5)$ are associated with three atmospheric regions that are separated by the two cloud layers, whereas $I_{i}^{\downarrow}$ and $I_{i}^{\uparrow}(i=2,4)$ are associated with the contributions from the cloud layers themselves. In the following discussion, the upward TOA radiance is formulated for a nadir-viewing space-borne instrument. The radiance for an off-nadir viewing configuration is a simple extension. Relatively strong absorption by clouds and the atmosphere in the IR ensures that the second and higher orders of reflections between the surface and the low-level cloud, and between the two cloud layers, are much smaller than their first-order counterparts and may be omitted without significant impact. In the following analysis, radiances are denoted as I and transmittances are denoted as $\Gamma$; both are spectral quantities but for clarity their spectral dependence is not made explicit.

The upward radiance at the top of the atmosphere is given by:

$$
I_{\text {top }}=I_{a}+I_{b}+I_{c}+I_{d},
$$




$$
\begin{aligned}
& \mathrm{I}_{\mathrm{a}}=\varepsilon_{\mathrm{s}} \mathrm{B}\left(\mathrm{T}_{\mathrm{s}}\right) \Gamma_{1} \Gamma_{2} \Gamma_{3} \Gamma_{4} \Gamma_{5}+\mathrm{I}_{1}^{\uparrow} \Gamma_{2} \Gamma_{3} \Gamma_{4} \Gamma_{5}+\mathrm{I}_{2}^{\uparrow} \Gamma_{3} \Gamma_{4} \Gamma_{5}+\mathrm{I}_{3}^{\uparrow} \Gamma_{4} \Gamma_{5}+\mathrm{I}_{4}^{\uparrow} \Gamma_{5}+\mathrm{I}_{5}^{\uparrow}, \\
& \mathrm{I}_{\mathrm{b}}=\Gamma_{1} \Gamma_{2} \Gamma_{3} \Gamma_{4} \Gamma_{5} \mathrm{r}_{\mathrm{s}}\left(\mathrm{I}_{1}^{\downarrow}+\mathrm{I}_{2}^{\downarrow} \Gamma_{1}+\mathrm{I}_{3}^{\downarrow} \Gamma_{1} \Gamma_{2}+\mathrm{I}_{4}^{\downarrow} \Gamma_{1} \Gamma_{2} \Gamma_{3}+\mathrm{I}_{5}^{\downarrow} \Gamma_{1} \Gamma_{2} \Gamma_{3} \Gamma_{4}\right), \\
& \mathrm{I}_{\mathrm{c}}=\Gamma_{3} \Gamma_{4} \Gamma_{5} \mathrm{r}_{\mathrm{L}}\left(\mathrm{I}_{3}^{\downarrow}+\mathrm{I}_{4}^{\downarrow} \Gamma_{3}+\mathrm{I}_{5}^{\downarrow} \Gamma_{3} \Gamma_{4}\right), \\
& I_{d}=\Gamma_{5} r_{H} I_{5}^{\downarrow},
\end{aligned}
$$

where $\Gamma_{1}, \Gamma_{3}$ and $\Gamma_{5}$ indicate transmittances of clear-sky atmospheric regions separated by the two cloud layers (as shown in Fig.1), while $\Gamma_{2}$ and $\Gamma_{4}$ are the transmittances associated with the low-level cloud, and high-level cloud, respectively. The quantities $r_{s}, r_{L}$ and $r_{H}$ represent the reflectance of the surface, low-level cloud, and high-level cloud, respectively, and $\varepsilon_{s}$ and $B\left(T_{s}\right)$ are the surface emissivity and the value of the Planck function at the surface temperature $T_{s}$, respectively. In Eqs. (3)-(6), $I_{a}$ represents the direct transmission of the thermal emission from the surface and the five atmospheric regions shown in Fig.1; $\mathrm{I}_{\mathrm{b}}$ represents the contribution from the reflection of the downward radiance at the surface; $I_{c}$ represents the reflection of downward radiance at the top of the low-level cloud; and $I_{d}$ represents the contribution from the reflection of the downward radiance at the top of the high-level cloud. The radiances in Eqs. (3)-(6) are given by:

$$
\begin{aligned}
& I_{1}^{\uparrow}=I_{1}^{\downarrow}=\int_{z_{s}}^{z_{i}} B\left(T_{z}\right) \frac{d \Gamma(z)}{d z} d z, \\
& I_{2}^{\uparrow}=I_{2}^{\downarrow}=\varepsilon_{2} B\left(T_{z_{2}}\right), \\
& I_{3}^{\uparrow}=I_{3}^{\downarrow}=\int_{z_{i+1}}^{z_{j}} B\left(T_{z}\right) \frac{d \Gamma(z)}{d z} d z, \\
& I_{4}^{\uparrow}=I_{4}^{\downarrow}=\varepsilon_{4} B\left(T_{z_{4}}\right),
\end{aligned}
$$




$$
I_{5}^{\uparrow}=I_{5}^{\downarrow}=\int_{z_{j+1}}^{z_{\infty}} B\left(T_{z}\right) \frac{d \Gamma(z)}{d z} d z
$$

where the low and high clouds are defined between $z_{i}$ and $z_{i+1}$, and $z_{j}$ and $z_{j+1}$ (see Fig.1). $\varepsilon_{2}$ and $\varepsilon_{4}$ represent cloud emissivities of two cloud layers at their effective heights of $z_{2}$ and $z_{4}$, respectively. The radiances in Eqs. (7)-(11) are computed from a clear-sky RT model that is incorporated into FIRTM2. An important point to note is that in FIRTM2 the treatment of the reflection of radiation by clouds and the surface is highly simplified. Isotropic downward radiation fields at the cloud top and at the surface are implicitly assumed in the derivation of $\mathrm{I}_{\mathrm{b}}$, $I_{c}$, and $I_{d}$ in Eqs. (4)-(6). For example, the reflected radiance at the top of the high-level cloud is given by:

$$
I(\mu)=\frac{1}{\pi} \int_{0}^{2 \pi} \int_{0}^{1} R_{H}\left(\mu, \varphi, \mu^{\prime}, \varphi^{\prime}\right) I\left(-\mu^{\prime}, \varphi^{\prime}\right) \mu^{\prime} d \mu^{\prime} d \varphi^{\prime}
$$

where $I\left(-\mu^{\prime}, \varphi^{\prime}\right)$ is the downward radiance at the cloud top and $R_{H}\left(\mu, \varphi, \mu^{\prime}, \varphi^{\prime}\right)$ is cloud bidirectional reflection function [18-20]. With the assumption of the isotropy of radiation field, the reflected radiance at the top of the high cloud is given by

$$
I(\mu)=I_{5}^{\downarrow} r_{H}(\mu)
$$

where

$$
r_{H}(\mu)=\frac{1}{\pi} \int_{0}^{2 \pi} \int_{0}^{\pi / 2} R_{H}\left(\mu, \varphi, \mu^{\prime}, \varphi^{\prime}\right) \mu^{\prime} d \mu^{\prime} d \varphi^{\prime}
$$

The use of a pre-computed table of cloud reflectance and transmittance values, i.e. $\mathrm{r}_{\mathrm{L}}, \mathrm{r}_{\mathrm{H}}, \Gamma_{2}$, and $\Gamma_{4}$ in Eqs. (3)-(6), substantially increases the computational efficiency of FIRTM2. The look-up table for water clouds involved in FIRTM2 is the same as that used in FIRTM1. For ice clouds, we use a mixture of several ice particle habits, following Baum et al. 
[21] and King et al. [23]. Specifically, it is assumed that for those ice clouds where the maximum dimension of an ice particle is smaller than $70 \mu \mathrm{m}$, the cloud comprises $50 \%$ bullet rosettes, $25 \%$ hollow columns, and $25 \%$ plates. For clouds comprised of particles larger than $70 \mu \mathrm{m}$, the assumption is that bullet rosettes and aggregates dominate the particle size distribution, specifically $30 \%$ aggregates, $30 \%$ bullet rosettes, $20 \%$ hollow columns, and $20 \%$ plates. The single-scattering properties of non-spherical ice crystals used in this study are those computed by Yang et al. [24]. Following Foot [25], Baum et al. [22] and King et al. [23]), we define the effective particle size for a given size distribution as follows:

$$
D_{e}=\frac{3 \int_{L_{\min }}^{L_{\max }}\left[\sum_{i=1}^{N} f_{i}(L) V_{i}(L)\right] n(L) d L}{2 \int_{L_{\min }}^{L_{\max }}\left[\sum_{i=1}^{N} f_{i}(L) A_{i}(L)\right] n(L) d L},
$$

where $L$ is the maximum dimension of an individual ice particle; $L_{\min }$ and $L_{\max }$ are the minimum and maximum particle sizes, respectively; $A_{i}(L)$ and $V_{i}(L)$ are the projected area and volume of the particle where index $i$ denotes ice particle habit; and $n(L)$ and $f_{i}(L)$ are the given particle size and habit distributions, The effective particle size defined in Eq. (1) reduces to that defined by Hansen and Travis [26] for water droplets.

Given a radiance, the brightness temperature can be computed from an inversion of the Planck function as follows:

$$
T_{B}=\frac{1.4385 v}{\ln \left(1+\frac{1.1911 \times 10^{-8} v^{3}}{I_{t o p}}\right)},
$$

where $v$ is the channel spectral wavenumber. Figure 2 shows the FIRTM2 calculated upward brightness temperatures for three different cloudy conditions. Fig.2a shows results of a singlelayered ice cloud located at the height of $12 \mathrm{~km}$ with an optical thickness of 1 and an effective 
particle size of $40 \mu \mathrm{m}$. Fig. $2 \mathrm{~b}$ shows results of a single-layered water cloud located at a height of $2 \mathrm{~km}$ with an optical thickness of 5 and an effective size of $10 \mu \mathrm{m}$. Fig.2c shows results for a multilayered cloud case where an ice cloud layer overlies a water cloud layer, in which the ice cloud is at a height of $12 \mathrm{~km}$ with an optical thickness of 1 and an effective size of $50 \mu \mathrm{m}$, and the water cloud located at $2 \mathrm{~km}$ with an optical thickness of 5 and an effective size of $10 \mu \mathrm{m}$.

\section{Accuracy Estimation of FIRTM2}

To estimate the accuracy of FIRTM2, we compare the top-of-atmosphere (TOA) brightness temperatures computed from FIRTM2 to those from DISORT for various atmospheric conditions. Figure 3 shows the brightness temperature (BT) differences (BTD) in terms of the differences between the BT from FIRTM2 minus the BT from DISORT, henceforth referred to simply as BTD (F-D). It is evident from Fig.3 that FIRTM2 is quite accurate, as the BTD (F-D) values are generally less than $0.5 \mathrm{~K}$. The overall feature shown in Fig. 3 is that FIRTM2 slightly overestimates the TOA brightness temperatures.

Figure 4 shows the BTD(F-D) values for ice clouds in the LW band (panels, a, b, and c) and in the SMW band (panels d, e, and f) for various effective particle sizes. The uppermost two panels in Fig. 4 are for a case when an ice cloud is located at $15 \mathrm{~km}$ above the surface; the middle two panels are for a case when the height of an ice cloud is $10 \mathrm{~km}$; and the bottom panels are for a case when the cloud height is $5 \mathrm{~km}$. For the LW band, the BTD(F-D) values monotonically increase with the ice cloud optical thickness. The effect of the particle size on the BTD(F-D) values is not significant as evidenced by panels (a), (b) and (c). For the SMW band, the BTD(F-D) values depend strongly on the particle size and cloud height. In particular, the BTD(F-D) distribution is quite different for small and large particle sizes when cloud is located 
high $(>10 \mathrm{~km})$ in the atmosphere. In general, the BTD(F-D) values for the LW band are less than $0.5 \mathrm{~K}$ if the ice cloud optical thickness is less than 5, whereas the BTD(F-D) values for SMW are less than $1 \mathrm{~K}$. For cases with large particle sizes or low cloud heights, the BTD(F-D) values are substantially smaller.

Figure 5 shows the BTD(F-D) values for water cloud optical thicknesses up to 100 and cloud heights of 1,2 , and $3 \mathrm{~km}$. Asymptotic values are reached when the optical thickness is larger than 50 for both the LW and the SMW band. It is evident from Fig. 5 that the accuracy of FIRTM2 increases with decreasing cloud height. The BTD(F-D) values at both bands are less than $0.8 \mathrm{~K}$ except at LW band when cloud is at a height of $3 \mathrm{~km}$.

Figure 6 shows BTD(F-D) values for cases involving two cloud layers. As a canonical simulation by FIRTM2, a water cloud layer is fixed with an optical thickness of 50 and an effective size of $10 \mu \mathrm{m}$ is defined at $2 \mathrm{~km}$; whereas ice cloud properties vary. The BTD(F-D) values depend on the optical thickness of the ice cloud. For the LW band, the BTD(F-D) values are not sensitive to the effective size of ice crystals. For the SMW band, BTD(F-D) values increase for high $(>10 \mu \mathrm{m})$ ice clouds with small ice crystals $(\mathrm{De}=10$ and $20 \mu \mathrm{m})$. In general, the $\mathrm{BTD}(\mathrm{F}-\mathrm{D})$ values are less than $1 \mathrm{~K}$.

\section{Sensitivity study with Jacobians}

The Jacobians are the partial derivatives of the brightness temperature (corresponding to the TOA radiance) with respect to cloud optical thickness and with respect to cloud effective particle size. The FIRTM2 provides an efficient way to compute Jacobians based upon their 
analytical formulation. From Eq. (16), the partial derivative of the brightness temperature with respect to the $\tau_{v i s}$ and $D e$ can be written as:

$$
\begin{aligned}
& \frac{\partial T_{B}}{\partial \tau_{v i s}}=\frac{1.7124 \times 10^{-8} v^{4}}{\left(I_{\text {top }}^{2}+1.1911 \times 10^{-8} v^{3} I_{\text {top }}\right)\left[\ln \left(1+\frac{1.1911 \times 10^{-8} v^{3}}{I_{\text {top }}}\right)\right]^{2}} \frac{\partial I_{\text {top }}}{\partial \tau_{v i s}}, \\
& \frac{\partial T_{B}}{\partial D e}=\frac{1.7124 \times 10^{-8} v^{4}}{\left(I_{\text {top }}^{2}+1.1911 \times 10^{-8} v^{3} I_{\text {top }}\right)\left[\ln \left(1+\frac{1.1911 \times 10^{-8} v^{3}}{I_{\text {top }}}\right)\right]^{2}} \frac{\partial I_{\text {top }}}{\partial D e},
\end{aligned}
$$

where the partial derivative of $I_{t o p}$ with respect to $\tau_{v i s}$ and $D e$ can be derived from Eq. (2) as follows:

$$
\begin{aligned}
& \frac{\partial I_{t o p}}{\partial \tau_{v i s}}=\left(\varepsilon_{s} B\left(T_{s}\right) \Gamma_{1}+I^{\uparrow}\right) \Gamma_{3} \Gamma_{5}\left(\Gamma_{4} \frac{\partial \Gamma_{2}}{\partial \tau_{v i s}}+\Gamma_{2} \frac{\partial \Gamma_{4}}{\partial \tau_{v i s}}\right)+\left(I_{2}^{\uparrow} \Gamma_{3}+I_{3}^{\uparrow}\right) \Gamma_{5} \frac{\partial \Gamma_{4}}{\partial \tau_{v i s}} \\
& \quad+r_{0} \Gamma_{1} \Gamma_{3} \Gamma_{5}\left[\left(I_{1}^{\downarrow}+I_{2}^{\downarrow} \Gamma_{2}\right)\left(\Gamma_{4} \frac{\partial \Gamma_{2}}{\partial \tau_{v i s}}+\Gamma_{2} \frac{\partial \Gamma_{4}}{\partial \tau_{v i s}}\right)+\left(I_{3}^{\downarrow}+I_{4}^{\downarrow} \Gamma_{3}\right) \Gamma_{1} \Gamma_{2}\left(2 \Gamma_{4} \frac{\partial \Gamma_{2}}{\partial \tau_{v i s}}+\Gamma_{2} \frac{\partial \Gamma_{4}}{\partial \tau_{v i s}}\right)\right] \\
& +2 I_{5}^{\downarrow} \Gamma_{1} \Gamma_{2} \Gamma_{3} \Gamma_{4}\left(\Gamma_{4} \frac{\partial \Gamma_{2}}{\partial \tau_{v i s}}+\Gamma_{2} \frac{\partial \Gamma_{4}}{\partial \tau_{v i s}}\right)+\left(I_{3}^{\downarrow}+I_{4}^{\downarrow} \Gamma_{3}\right) \Gamma_{3} \Gamma_{5}\left(\Gamma_{4} \frac{\partial \Gamma_{2}}{\partial \tau_{v i s}}+\Gamma_{2} \frac{\partial \Gamma_{4}}{\partial \tau_{v i s}}\right) \\
& \quad+I_{5}^{\downarrow} \Gamma_{3} \Gamma_{4}\left(\Gamma_{4} \frac{\partial \Gamma_{2}}{\partial \tau_{v i s}}+2 \Gamma_{2} \frac{\partial \Gamma_{4}}{\partial \tau_{v i s}}\right)+I_{5}^{\downarrow} \Gamma_{5} \frac{\partial \Gamma_{4}}{\partial \tau_{v i s}}
\end{aligned}
$$




$$
\begin{aligned}
& \frac{\partial I_{t o p}}{\partial D e}=\left(\varepsilon_{s} B\left(T_{s}\right) \Gamma_{1}+I^{\uparrow}\right) \Gamma_{3} \Gamma_{5}\left(\Gamma_{4} \frac{\partial \Gamma_{2}}{\partial D e}+\Gamma_{2} \frac{\partial \Gamma_{4}}{\partial D e}\right)+\left(I_{2}^{\downarrow} \Gamma_{3}+I_{3}^{\downarrow}\right) \Gamma_{5} \frac{\partial \Gamma_{4}}{\partial D e} \\
& +r_{0} \Gamma_{1} \Gamma_{3} \Gamma_{5}\left[\left(I_{1}^{\downarrow}+I_{2}^{\downarrow} \Gamma_{2}\right)\left(\Gamma_{4} \frac{\partial \Gamma_{2}}{\partial D e}+\Gamma_{2} \frac{\partial \Gamma_{4}}{\partial D e}\right)+\left(I_{3}^{\downarrow}+I_{4}^{\downarrow} \Gamma_{3}\right) \Gamma_{1} \Gamma_{2}\left(2 \Gamma_{4} \frac{\partial \Gamma_{2}}{\partial D e}+\Gamma_{2} \frac{\partial \Gamma_{4}}{\partial D e}\right)\right] \\
& +2 I_{5}^{\downarrow} \Gamma_{1} \Gamma_{2} \Gamma_{3} \Gamma_{4} \frac{\partial \Gamma_{2}}{\partial D e}+\left(I_{3}^{\downarrow}+I_{4}^{\downarrow} \Gamma_{3}\right) \Gamma_{3} \Gamma_{5}\left(\Gamma_{4} \frac{\partial \Gamma_{2}}{\partial D e}+\Gamma_{2} \frac{\partial \Gamma_{4}}{\partial D e}\right) \\
& +I_{5}^{\downarrow} \Gamma_{3} \Gamma_{4}\left(\Gamma_{4} \frac{\partial \Gamma_{2}}{\partial \tau_{v i s}}+2 \Gamma_{2} \frac{\partial \Gamma_{4}}{\partial \tau_{v i s}}\right)+I_{5}^{\downarrow} \Gamma_{5} \frac{\partial \Gamma_{4}}{\partial D e} .
\end{aligned}
$$

To solve (19) and (20), a total of eight sets of partial derivatives of cloud reflectance and transmittance $\left(r \& \Gamma\right.$ ) with respect to $\tau_{v i s}$ and $D e$ are derived in FIRTM2 by differentiating the $r$ $\& \Gamma$ lookup-tables with small perturbations to $\tau_{v i s}$ and $D e$ at their specific values. As an example, Fig. 7 shows the Jacobian $\left(\partial T_{B} / \partial \tau_{v i s}\right)$ for a single-layered ice cloud for various effective particle sizes where a $1 \%$ perturbation is applied to unit visible optical thickness cloud at an altitude of $12 \mathrm{~km}$.

Figure 8 shows the sensitivity of the derivative of the TOA-radiance-equivalent brightness temperature to the effective particle sizes for three values of ice cloud optical thickness: $\tau_{\text {vis }}=1$ (panel a), 6 (panel b), and 10 (panel c). For a large optical thickness (i.e., the case shown in panel c), the derivative of the brightness temperature with respect to the optical thickness $\partial \mathrm{T}_{\mathrm{b}} / \partial \tau_{\mathrm{vis}}$ as a function of the wavelength is not sensitive to the effective particle size, as the variations of $\partial \mathrm{T}_{\mathrm{b}} / \partial \tau_{\mathrm{vis}}$ are quite small in this case. The sensitivity of $\partial \mathrm{T}_{\mathrm{b}} / \partial \tau_{\mathrm{vis}}$ to the effective particle size increases with the decrease of the optical thickness. An interesting point to note is that the sensitivity is minimal at wavenumbers near $890 \mathrm{~cm}^{-1}$. This can be a useful feature for retrieving cloud properties. For example, the cloud optical thickness can be estimated from 
minimizing the difference between the simulated and observed radiances. Given an estimate for the optical thickness, the effective particle size can be estimated from the slope of the spectrum between 800 to $900 \mathrm{~cm}^{-1}$ (e.g., the studies reported by Huang et al. [27]; Wei et al. [9]). The estimates of both effective particle size and optical thickness can be improved further through iteration until a convergent solution is found.

\section{Summary}

A fast infrared radiative transfer model (FIRTM2) is developed to compute the TOA radiance for overlapping clouds, and can be applied to three atmospheric conditions: a singlelayered ice cloud, a single-layered liquid water cloud, and a multilayered cloud (e.g., an ice cloud layer over a liquid water cloud). In FIRTM2, the cloud properties are based upon a set of precomputed look-up tables of cloud reflectance and transmittance values. The background atmospheric optical thickness due to gaseous absorption are computed from a clear-sky RT model that can be either a fast clear-sky RT model or a line-by-line model, depending on the desired application. In FIRTM2, Jacobians (i.e., the derivatives of the TOA brightness temperature with respect to the optical thickness and effective particle size) are also computed.

Extensive comparisons of FIRTM2 with DISORT have been carried out to assess the numerical accuracy of FIRTM2. For a single-layered ice cloud, the brightness temperature differences between FIRTM2 and DISORT $[\mathrm{BTD}(\mathrm{F}-\mathrm{D})]$ in the LW band is better than $0.5 \mathrm{~K}$ when the optical thickness is less than 5. In the SMW band, the BTD(F-D) values are less than $0.8 \mathrm{~K}$ except for very high clouds comprised primarily of small particles. For a single-layered water cloud, the BTD(F-D) values for both the LW and SMW bands are better than $0.8 \mathrm{~K}$ except for the case of the LW band with a cloud located at $3 \mathrm{~km}$. For a multilayered cloud case 
consisting of an ice cloud over a water cloud, the BTD(F-D) values in both bands are typically less than $0.8 \mathrm{~K}$, except again for the case of small particles at high altitude $(>10 \mathrm{~km})$.

\section{Acknowledgment}

This work is supported by the GIFTS-IOMI MURI Project, and partially by the National Science Foundation (ATM-0239605) and the NASA Radiation Sciences Program (NNG04GL24G). 


\section{References}

[1] Aumann H. H., Chahine M. T., Gautier C., Goldberg M. D., Kalnay E., McMillin L. M., Revercomb H., Rosenkranz P. W., Smith W. L., Staelin D. H., Strow L., and Susskind J., AIRS/AMSU/HSB on the Aqua mission: Design, Science Objectives, Data products, and processing system, IEEE Trans. Geosci. Remote Sensing, 2003; 41: 253-264.

[2] Zhou D. K., Smith W. L., Li J., Howell H. B., Cantwell G. W., Larar A. M., Knuteson R. O., Tobin D. C., Revercomb H. E., and Mango S. A., Thermodynamic product retrieval methodology and validation for NAST-I, Appl. Opt., 2002; 41: 6957-6967.

[3] Strow L. L., Motteler H. E., Benson R. G., Hannon S. E., and SouzaMachado S., Fast computation of monochromatic infrared atmospheric transmittances using compressed look-up tables, J. Quant. Spectrosc. Radiat. Transf., 1998; 59: 481-493.

[4] Moncet J. L., and Clough S. A., Accelerated monochromatic radiative transfer for scattering atmospheres: Application of a new model to spectral radiance observations, J. Geophys. Res, 1997; 102: 21,853-21,866.

[5] Rathke C., and Fischer J., Retrieval of cloud microphysical properties from thermal infrared observations by a fast interative radiance fitting method, J. Atmos. Oceanic Technol. 2000; 17: 1509-1524.

[6] Rathke C., and Fishcher J., Evaluation of four approximate methods for calculating infrared radiances in cloudy atmospheres, J. Quant. Spectrosc. Radiat. Transfer, 2002; 75: 297-321. 
[7] Stamnes K., Tsay S., Wiscombe W., and Jayaweera K., Numerically stable algorithm for discrete-ordinate-method radiative transfer in multiple scattering and emitting layered media, Applied Optics, 1988; 27 (12): 2502-2509.

[8] Fu Q., Liou K. N., Cribb M. C., Charlock T. P., and Grossman A., Multiple scattering parameterization in thermal infrared radiative transfer, J. Atmos. Sci., 1997; 54: 2799-2812.

[9] Wei H., Yang P., Li J., Baum B. A., Huang H., Platnick S., Hu Y., and Strow L., Retrieval of Semitransparent Ice Cloud Optical Thickness From Atmospheric Infrared Sounder (AIRS) Measurements, IEEE Trans. Geosci. Remote Sensing, 2004; 42: 2254-2267.

[10] Mishchenko M. I. and Travis L. D., Capabilities and limitations of a current FORTRAN implementation of the T-matrix method for randomly oriented rotationally symmetric scatterers, J. Quant. Spectrosc. Radiat. Transfer, 1998; 60: 309-324.

[11] Fu Q., Sun W. B., and Yang P., On model of scattering and absorption by cirrus nonsperical ice particles at thermal infrared wavelength, J. Atmos. Sci. 1999; 56: 2937-2947.

[12] Yee K. S., Numerical solution of initial boundary problems involving Maxwell's equations in isotropic media, IEEE Trans. Antennas Propagat. 1966; 14: 302-307.

[13] Taflove A. Computational Electrodynamics: The Finite-Difference Time-Domain Method, Artech House, Boston, Mass., 1995. 
[14] Yang P., and Liou K. N., Finite-difference time domain method for light scattering by small ice crystals in three-dimensional space, J. Opt. Soc. Amer., 1996; A13: 20272080.

[15] Sun W., Fu Q., and Chen Z., Finite-difference time-domain solution of light scattering by dielectric particles with perfectly matched layer absorbing boundary conditions, Appl. Opt. 1999; 38: 3141-3151.

[16] Yang, P., Kattawar G. W., Liou K.N., and Lu J. Q., Choice of Cartesian grid configurations for applying the finite-difference time domain method to electromagnetic scattering by dielectric particles. Appl. Opt., 2004; 43: 4611-4624.

[17] Yang P., and Liou K. N., Geometric-optics-integral-equation method for light scattering by nonspherical ice crystals, Appl. Opt. 1996; 35: 6568-6584.

[18] Stamnes, K., Reflection and transmission by a vertically inhomogeneous planetary atmosphere, Planet. Space Sci., 1982; 30: 727-732.

[19] Thomas, G. E., and Stamnes K., Radiative Transfer in the Atmosphere and Ocean, Cambridge University Press, Cambridge, UK, p. 517.

[20] Liou, K. N., An Introduction to Atmospheric Radiation (2 ${ }^{\text {nd }}$ edition), San Diego, 2002.

[21] Baum B. A., Harkey M. K., Frey R. A., Mace G. G., and Yang P., Nighttime Multilayered could detection using MODIS and ARM data, J. Appl. Meteor. 2003; 42: 905-919.

[22] Baum B. A., Kratz D. P., Yang P., Ou S. C., Hu Y., Soulen P., and Tsay S. C., Remote sensing of cloud properties using MODIS Airborne Simulator imagery during SUCCESS I: Data and models, J. Geophys. Res. 2000; 105: 11,767-11,780. 
[23] King M. D., Platnick S., Yang P., Arnold G. T., Gray M. A., Riedi J. C., Ackerman S. A., and Liou K. N., Remote sensing of liquid water and ice cloud optical thickness, and effective radius in the arctic: Application of air-born multispectral MAS data, J. Atmos. And Ocean Technol. 2004; 21: 857-875.

[24] Yang, P., Wei H., Huang H.-L., Baum B. A., Hu Y. X., Kattawar G. W., Mishchenko M. I., and Fu Q., Scattering and absorption property database for nonspherical ice particles in the near- through far-infrared spectral region, Appl. Opt. 2005; (submitted).

[25] Foot J. S., Some observations of the optical properties of clouds: II. Cirrus,” Q. J. R. Meteor. Soc. 1988; 114: 145-164.

[26] Hansen JE, Travis LD, Light scattering in planetary atmosphere. Space Sci. Rev 1974; 16: 527-610

[27] Huang, H.-L., Yang P., Wei H., Baum B.A., Hu Y. X., Atonelli P., and Ackerman S. A., Inference of ice cloud properties from high-spectral resolution infrared observations. IEEE Trans. Geosci. Remote Sens. 2004; 42: 842-852. 


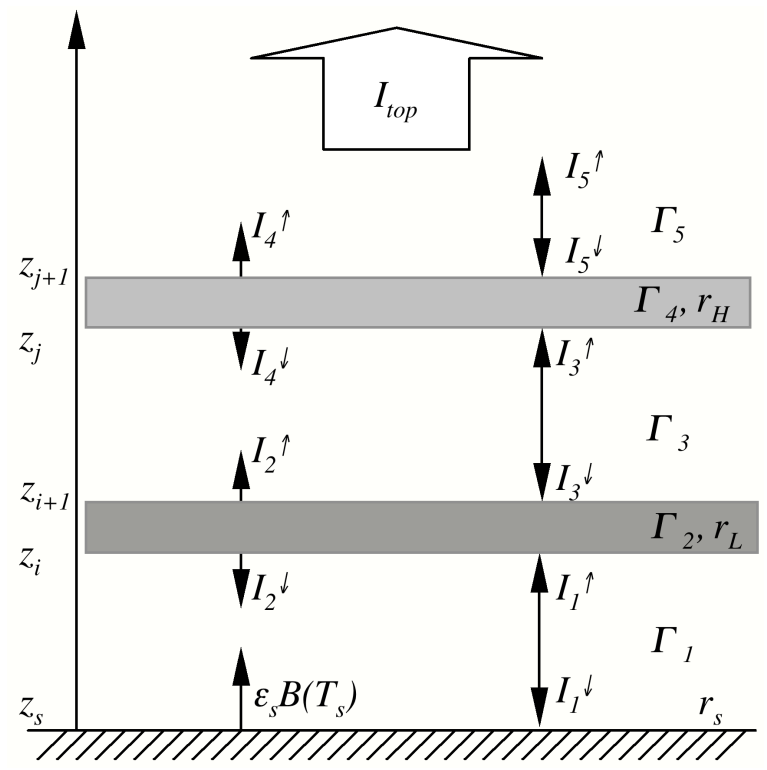

Fig. 1 Schematic configuration for a two-layer cloudy atmosphere and the decomposition of the atmosphere/cloud contributions to the upward radiance at the top of the atmosphere. 

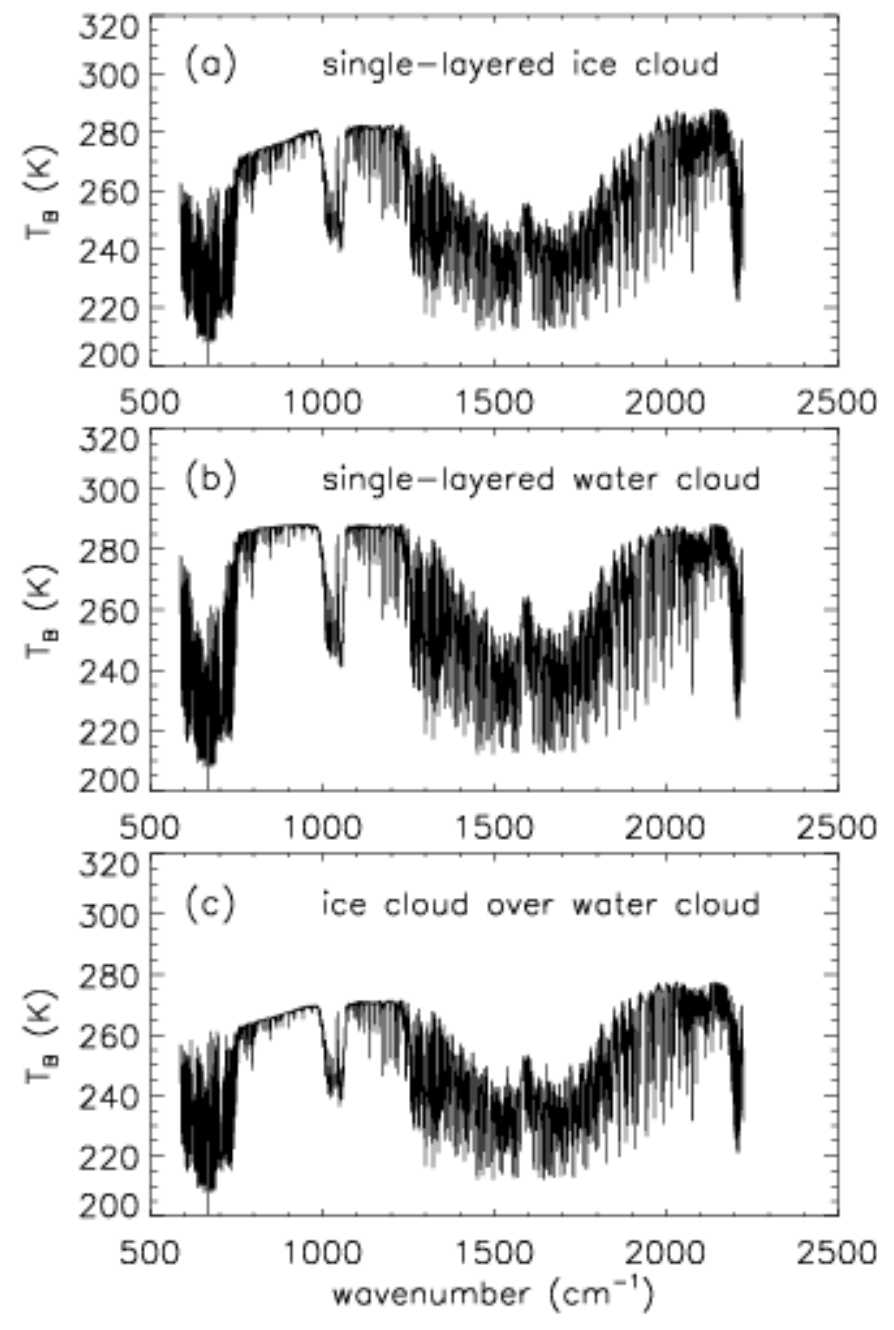

Fig. 2 The brightness temperatures corresponding to the TOA radiances computed from FIRTM2 for three atmospheric conditions: (a) a single-layered ice cloud at a height of 12 $\mathrm{km}\left(\tau_{v i s}=1, D e=40 \mu \mathrm{m}\right),(\mathrm{b})$ a single-layered water cloud at a height of $2 \mathrm{~km}\left(\tau_{v i s}=5, D e=\right.$ $10 \mu \mathrm{m})$, and (c) a cloud system with an ice cloud layer $\left(\tau_{v i s}=1, D e=50 \mu \mathrm{m}\right)$ at $12 \mathrm{~km}$ overlying a water cloud layer $\left(\tau_{v i s}=5, D e=10 \mu \mathrm{m}\right)$ at $2 \mathrm{~km}$. 

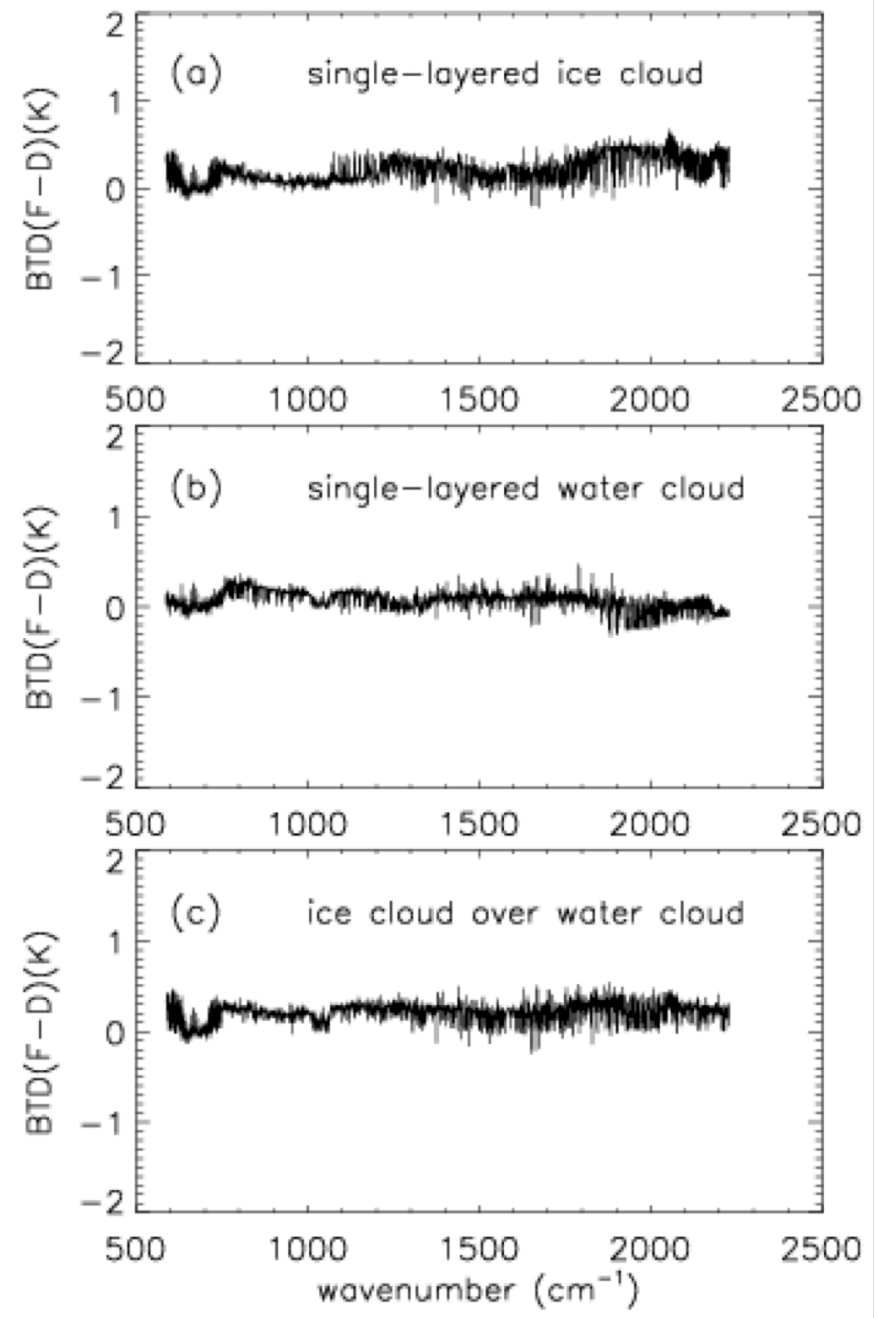

Fig 3. The brightness temperature differences between the FIRTM2 and DISORT solutions [BTD(F-D)] for the same atmospheric conditions as in Fig. 2. The brightness temperature differences shown here are defined as $\mathrm{BTD}=\mathrm{BT}_{\mathrm{FIRTM} 2}-\mathrm{BT}_{\mathrm{DISORT}}$. 

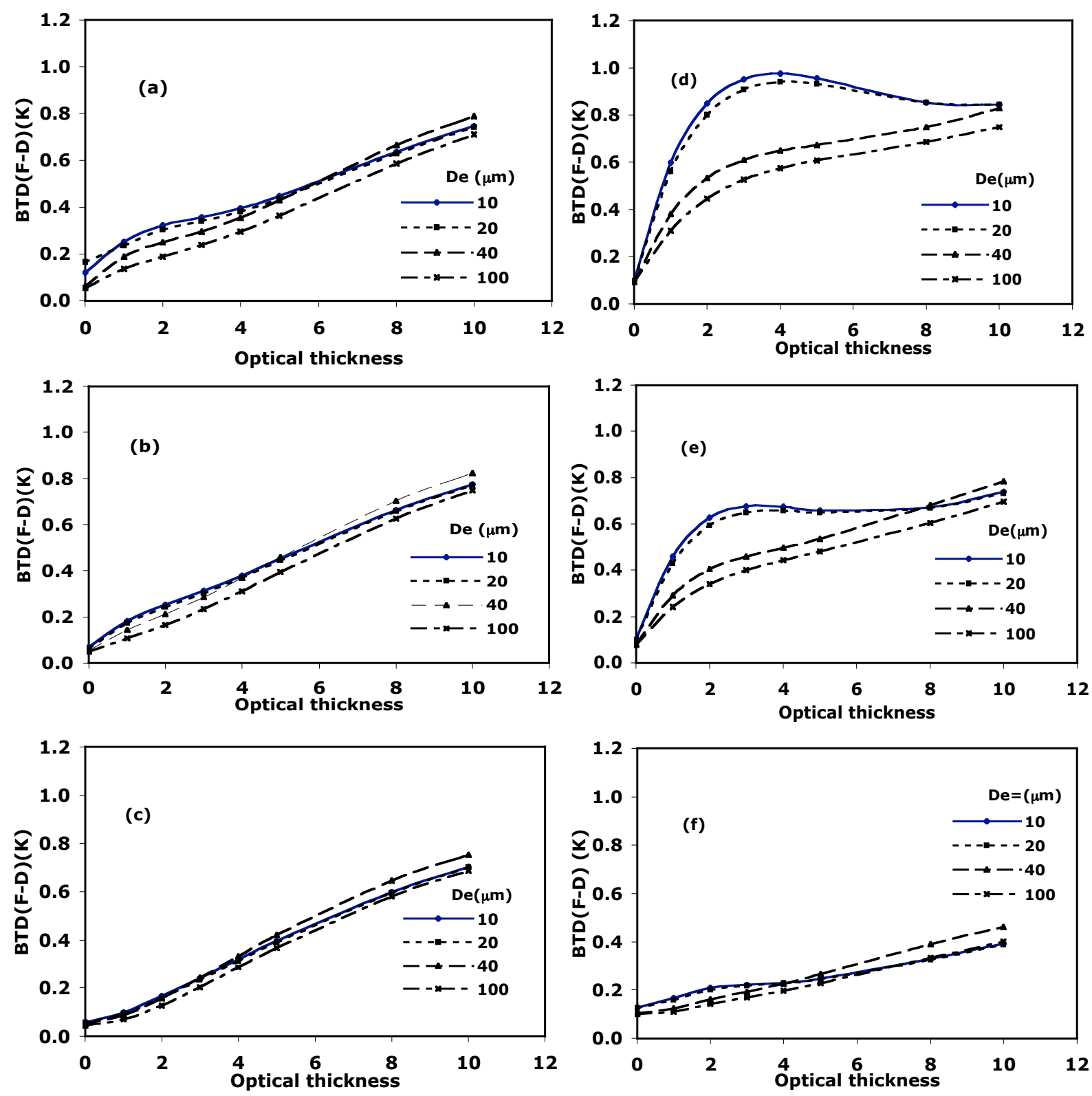

Fig. 4. The BTD(F-D) values for a single-layered ice cloud with respect to optical thickness, effective size, and cloud height. Panels (a), (b), and (c) are for the LW band; and panels (d), (e), and (f) are for the SMW band. Panels (a) and (d) are for clouds at $15 \mathrm{~km}$. Panels (b) and (e) are for clouds at $10 \mathrm{~km}$. Panels (c) and (f) are for clouds at $5 \mathrm{~km}$. 

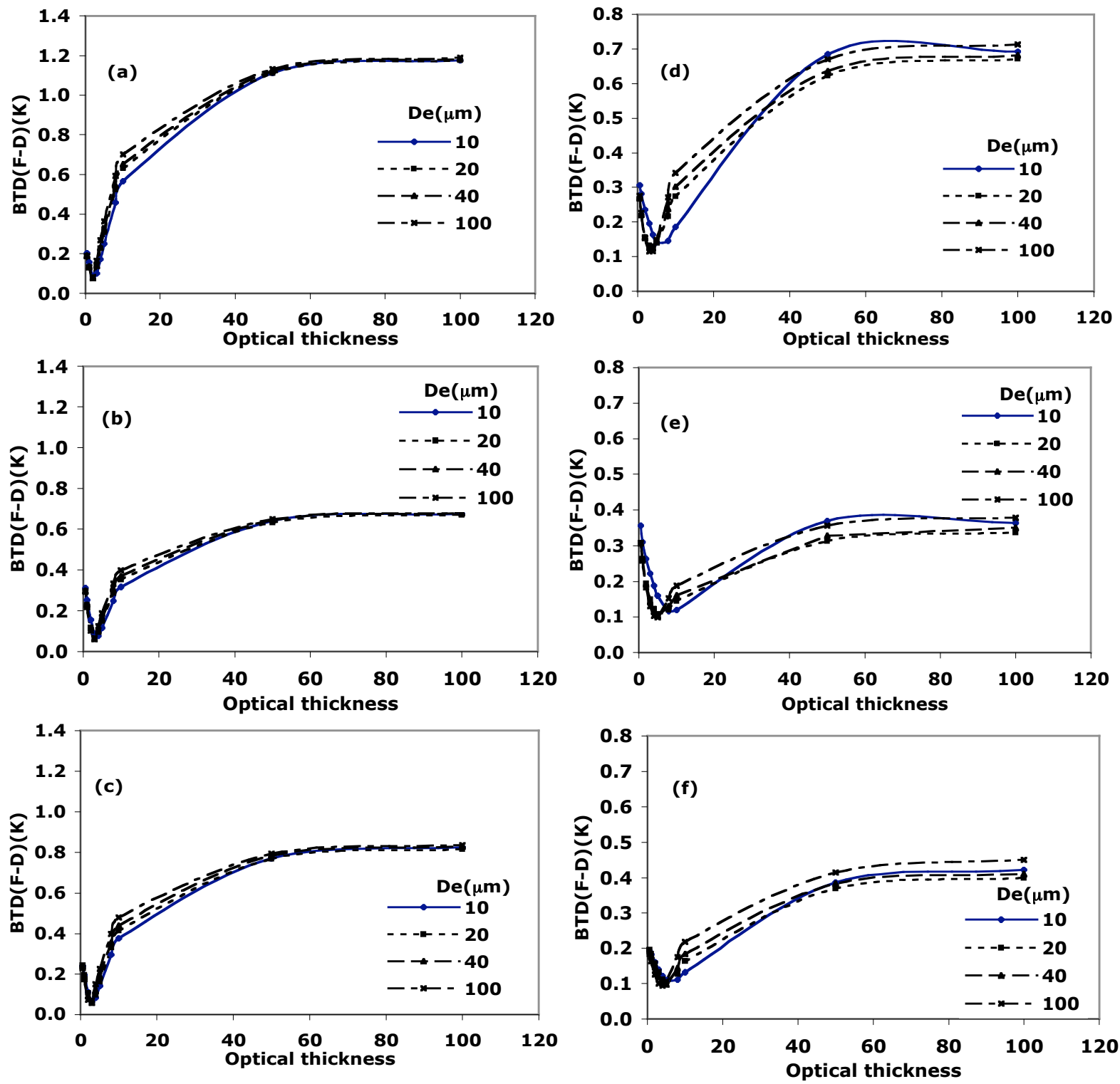

Fig. 5 Same as Fig.4 except for water clouds located at 3, 2, and $1 \mathrm{~km}$. 

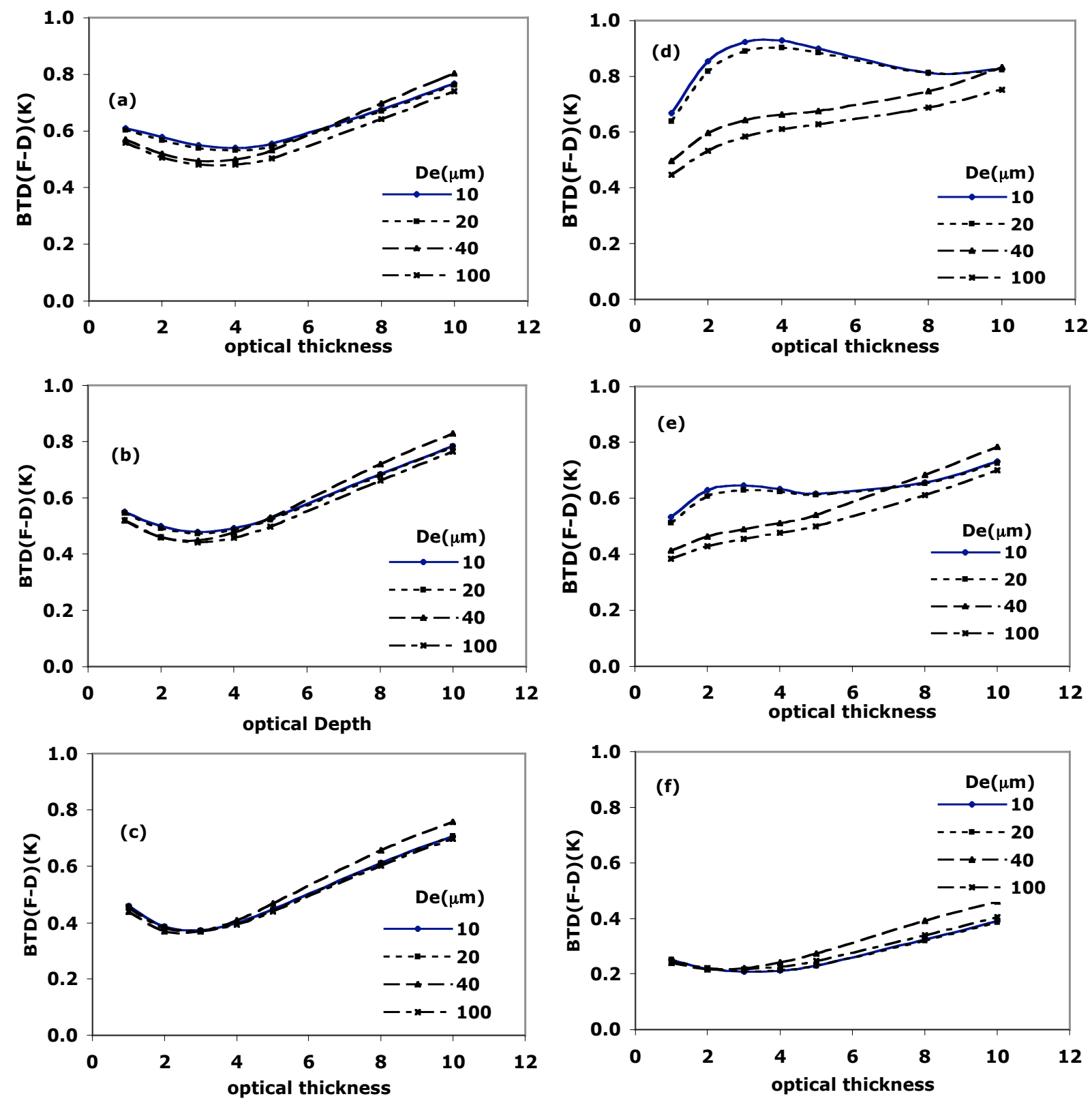

Fig. 6. Range of BTD(F-D) values for a case involving two cloud layers, with ice cloud overlying a water cloud. Panels (a), (b), and (c) are for the LW band; whereas panels (d), (e), and (f) are for the SMW band. An ice cloud is defined at $15 \mathrm{~km}$ for panels (a) and (d), $10 \mathrm{~km}$ for panels (b) and (e), and $5 \mathrm{~km}$ for panels (c) and (f). 


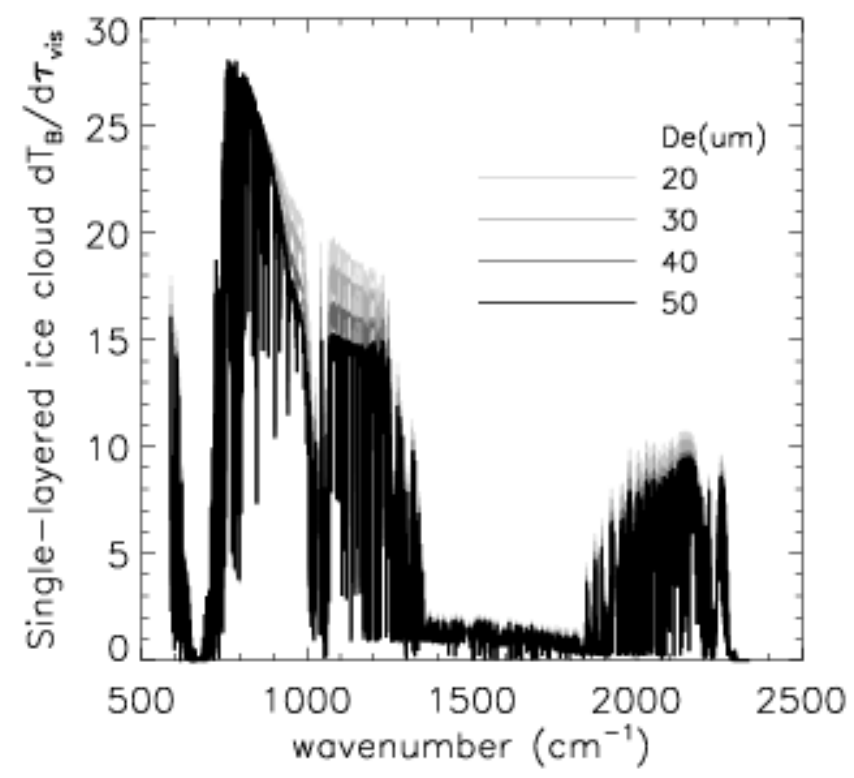

Fig. 7. The derivative of brightness temperature with respect to optical thickness for a singlelayered ice cloud located at a height of $12 \mathrm{~km}$ with an optical thickness $\tau_{\mathrm{vis}}=1$. 

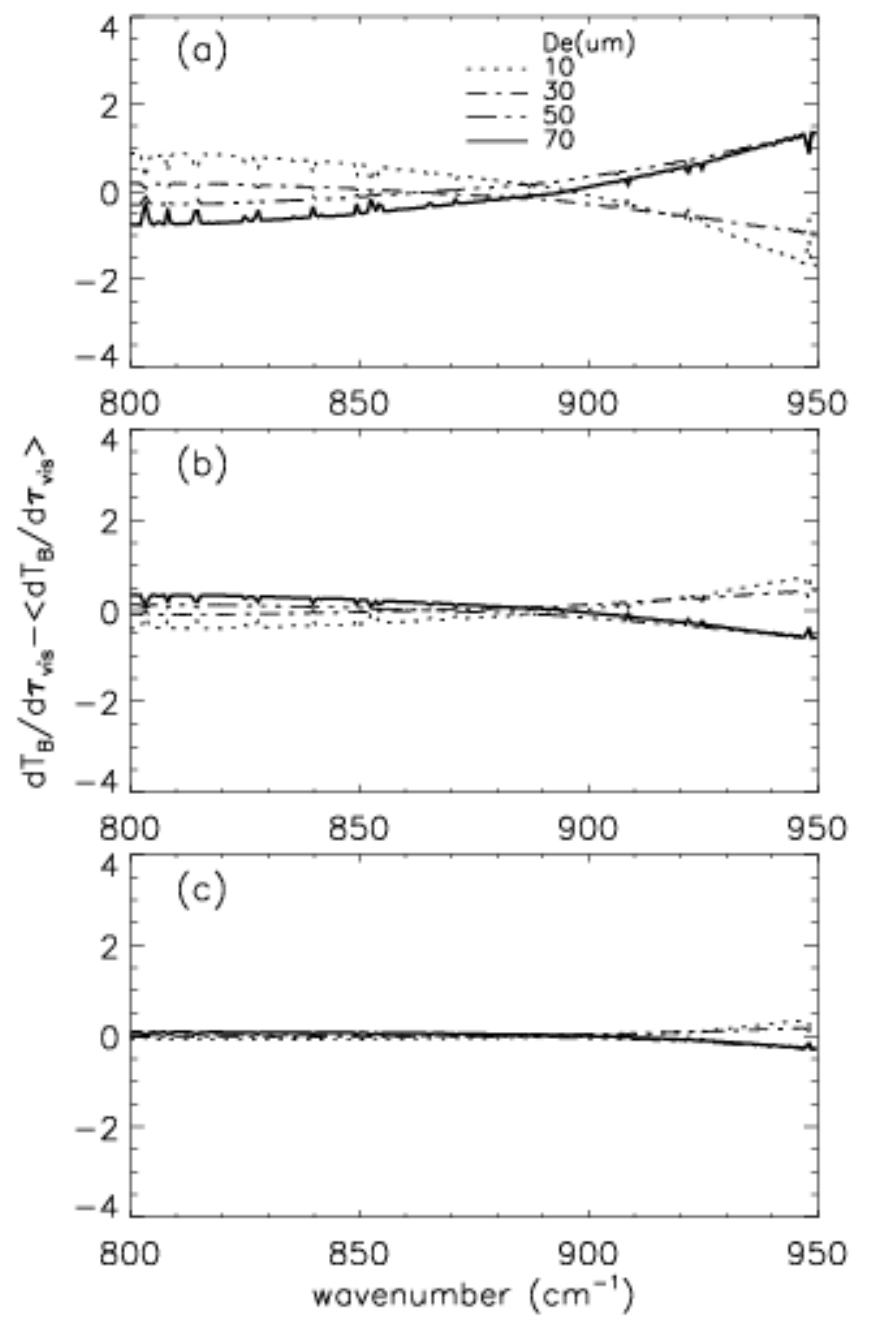

Fig. 8 The effect of ice cloud effective particle size on Jacobians $\left(\partial T_{B} / \partial \tau_{v i s}\right)$. Panel (a) $\tau_{v i s}=1$, (b) $\tau_{v i s}=6$, (c) $\tau_{v i s}=10$. In this simulation the ice cloud is located at $12 \mathrm{~km}$. 